\title{
A meta-analysis: neoadjuvant chemotherapy versus primary surgery in ovarian carcinoma FIGO stagelll and IV
}

Ma Dai-yuan*, Tan Bang-xian, Li Xian-fu, Zhou Ye-qin and Cai Hong-Wei

\begin{abstract}
Background: The purpose of the current study is to analyze the existing data comparing neoadjuvant chemotherapy with primary debulking surgery (PDS) in patients with advanced ovarian carcinoma.

Methods: Patients with stage IIIC and IV ovarian cancer were identified from articles in Medline, PubMed, Cochrane Library, and EMBASE database (1989 to February 2013). Two authors independently extracted the data. To assess the risk of bias of included literatures, Cochrane Collaboration's risk of bias tool was used. Meta-analysis on literatures was conducted by using RevMan 5.2 software.

Results: Two high-quality randomized controlled trials (RCTs) met the inclusion criteria. These multicenter trials randomized 1,220 women with stage IIIC/IV ovarian cancer to NACT or PDS followed by chemotherapy. There were no significant differences between the study groups with regard to overall survival (OS) (1,120 women; HR 0.98; $95 \% \mathrm{Cl} 0.85$ to 1.14) or progression-free survival (PFS) (1,120 women; HR 1.03; $95 \% \mathrm{Cl} 0.91$ to 1.16).

Conclusion: There was no statistical difference in median OS and PFS between the two treatment groups. With regard to selecting who will benefit from NACT, treatment should be tailored to the patient and should take into account respectability, age, histology, stage, and performance status.
\end{abstract}

Keywords: Ovarian carcinoma, Primary surgery, Neoadjuvant chemotherapy

\section{Review}

Introduction

An estimated 22,280 new cases of ovarian cancer are expected in the US in 2012 [1]. Ovarian cancer causes more deaths than any other cancer of the female reproductive system [1]. In most patients with ovarian carcinoma, the disease is diagnosed at an advanced stage and they usually have a very poor prognosis [2].

Primary surgical treatment of ovarian cancer has advantages in terms of diagnosis, staging, and tumor debulking [3,4]. The value of debulking surgery is well established in FIGO stage III epithelial ovarian cancer [5]. Most women will have widespread disease, therefore surgery alone does not cure the disease. Neoadjuvant chemotherapy (NACT) prior to surgical debulking proposes to increase the proportion of patients who may be

\footnotetext{
* Correspondence: mdylx@163.com

Department of Oncology, the First Affiliated Hospital of North Sichuan Medical College, Nanchong 637000, People's Republic of China
}

optimally cytoreduced, while decreasing surgical morbidity and mortality [6].

Several retrospective studies have shown that there was no difference in overall survival (OS) or progression-free survival (PFS) for patients with advanced ovarian cancer treated with neoadjuvant chemotherapy compared with primary debulking surgery (PDS) [7-11]. However, the result of a meta-analysis of Bristow and Chi [12] involving 835 patients suggested that NACT, compared with PDS, was associated with a worse OS and it was suggested that the definitive operative intervention should be undertaken as early in the treatment program as possible. But a more recent meta-analysis [13] of multiply central randomized trials concluded that survival was similar in patients treated with NACT followed by interval debulking surgery compared to primary debulking followed by chemotherapy and criticized the meta-analysis of Bristow and Chi [12].

Since randomized controlled trials (RCTs) are the 'gold standard' of evidence-based medical research, we hope 
that a review of randomized evidence may clarify what the benefits and risks are of using NACT for women with advanced ovarian cancer, compared with the standard treatment of PDS [13,14]. The purpose of the current study is to analyze the existing data comparing NACT with PDS in patients with advanced ovarian carcinoma which included more RCTs.

\section{Methods}

\section{Literature research}

We undertook computerized literature searches of MEDLINE, PubMed, Cochrane Library, and EMBASE databases, from their inception to February 2013. Search terms were 'ovarian carcinoma,' 'ovarian cancer', 'neoadjuvant chemotherapy', and 'primary surgery'. These terms were used in different combinations with each other. Appropriate references cited by the retrieved studies were also identified.

\section{Study selection}

Publications were selected for initial review if the research subjects were patients with International Federation of Gynecology and Obstetrics (FIGO) stage III and IV ovarian cancer who underwent NACT and PDS. In order to fully exclude the possibility of selection bias, only RCTs of NACT versus PDS were permitted. Duplicate publications or data were carefully reviewed by two of the authors and the larger (primary decision) or the most recent publication was included.

Data on trial size, patient characteristics (age, sex, AF duration, left ventricular size, left ventricular ejection fraction, and so on), procedure duration, and patient number of sinus rhythm maintenance without anti-arrhythmic drugs were extracted. Included studies were reviewed based on randomization, allocation concealment, blinding, loss of follow-ups, and ITT. The truths of the studies were divided into three grades according to Cochrane system evaluation handbook: Grade A, cases met all evaluated standards and had correct methodology, which had low risk of bias; Grade B, cases did not describe one or several standards, which had moderate risk of bias; Grade $\mathrm{C}$, cases had one or several standards incorrect, which had high risk of bias.

\section{Data extraction}

All the work of the literature search was independently reviewed by two authors to identify relevant trials that met the inclusion criteria and checked by an independent reviewer. Disparities were resolved by discussion. The following data were extracted from each study: (1) publication and trial characteristics, including the first author's name, publication year, study period, center, study design, and sample size; (2) clinical data, including patient data (gender, age, quality of life) and tumor data (histological characteristics and grade classifications); (3) data of outcome, including value of OS and PFS. All data were centrally reanalyzed and checked for inconsistencies. In this article, the primary endpoint was OS. Secondary endpoints were PFS. OS was defined as the time from distribution until death from any cause or to the last follow-up that was used as a date of censoring. PFS was defined as the time from distribution to relapse or death from any cause, whichever came first. Data for patients alive without progression were censored on the date of last follow-up.

\section{Statistical analysis}

Meta-analyses were carried out by one author using RevMan5.2. For survival outcomes (OS and PFS), the log

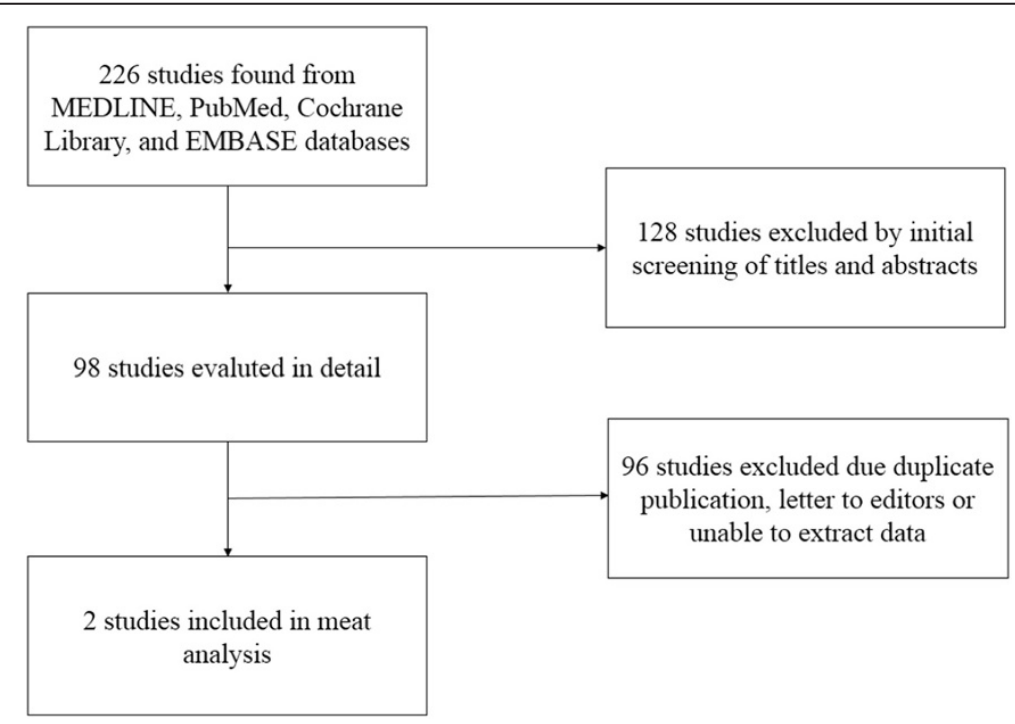

Figure 1 Process of study selection. 


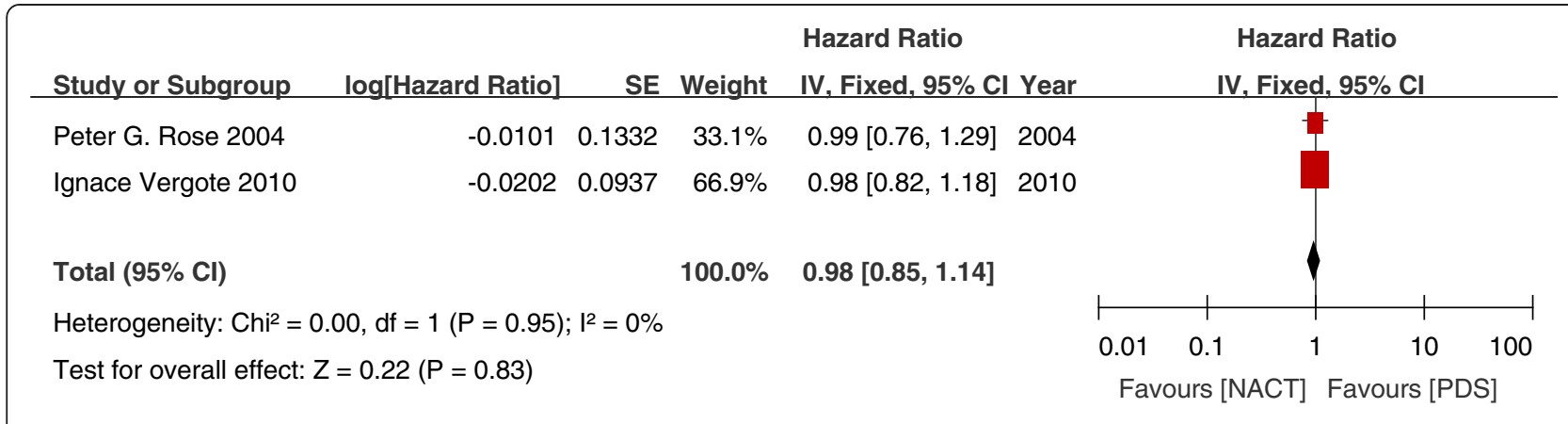

Figure 2 Overall survival outcome.

RRs from the various trials were combined in a metaanalysis using the Generic Inverse Variance facility of RevMan5.2. As a data analysis method, a fixed effect model was applied when the homogeneity between studies was verified. An I ${ }^{2}$ test was applied to determine between-study heterogeneity.

\section{Results}

Search result

The search process of the study is shown in Figure 1. A total of 226 publications were identified after initial search. The titles and abstracts of these articles were examined to exclude irrelevant trials. We also examined the reference lists of all relevant letters, editorials, and review articles. As a result, two articles possibly met the selection criteria (Peter G Rose et al., 2004; IgnaceVergote et al., 2010). Subsequently, the full text of these studies was examined thoroughly.

\section{Overall survival}

There was no significant difference in OS between the NACT and PDS groups (1,120 women; HR 0.98; $95 \%$ CI 0.85 to 1.14; Figure 2).

\section{Progression-free survival}

The existing data showed no significant difference in PFS between the NACT and PDS groups (670 women; HR 1.01; 95\% CI 0.87 to 1.17; Figure 3).

\section{Conclusion}

There was no statistical difference in median OS and PFS between the two treatment groups. With regard to selecting who will benefit from NACT, treatment should be tailored to the patient and should take into account respectability, age, histology, stage, and performance status.

\section{Discussion}

The clinical basis of aggressive cytoreductive surgery in the initial management of ovarian cancer is the significantly improved survival gained by those patients in whom optimal cytoreductive surgery was accomplished [14]. The presence of residual disease after surgery is one of the most adverse prognostic factors for survival. Therefore, although the definition of optimal cytoreduction has been modified over the last two decades, it is generally agreed that every attempt should be made to surgically resect as much disease as safely possible [15]. There are many hypothesized advantages to NACT in women who have advanced ovarian cancer that likely cannot be optimally cytoreduced. Chemotherapy can resolve pleural effusions and ascites and improve the patient's performance status prior to surgery $[16,17]$. There have been reports of subjective improvements in the sense of wellbeing and quality of life $[18,19]$. It can decrease tumor volume and increase respectability. Thus, patients may have less intraoperative blood loss, shorter operative times, less

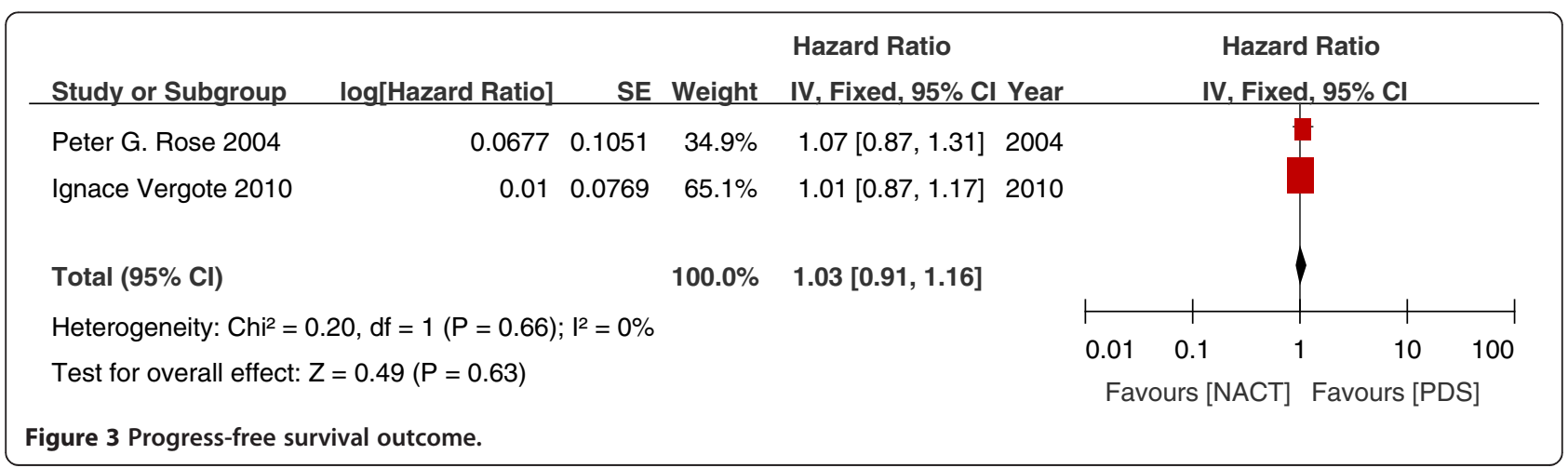


intensive care unit admissions, and shorter length of hospital stay $[4,14,19]$. These issues are particularly important for patients with medical co-morbidities and a low probability of cure.

Primary cytoreductive surgery is still the gold standard in the treatment of ovarian carcinoma [20]. NACT for advanced unresectable ovarian carcinoma led to the selection of a group of patients sensitive to chemotherapy, in whom secondary cytoreductive surgery can be achieved in a less aggressive manner. Also NACT prevents mutilating surgery in patient with a very poor prognosis either because of progressive disease or because of primary chemoresistance. These findings must be confirmed by a larger prospective study [21].

There are several limitations to this current study that must be considered in interpreting the data. This is a retrospective study, the limitations imposed by these attributes have to be borne in mind when interpreting or using the findings [22]. A standardized protocol to monitor chemotherapy response was not used in these patients thus potentially introducing lead-time bias, nor was our study designed to control for various second and further-line treatment regimens, which may have the ultimate impact on OS [23].

\section{Competing interests}

The authors declare that they have no competing interests.

\section{Authors' contributions}

DM independently searched references and took charge of data statistics and drafted the manuscript. BT and XL searched references and extracted the parameters from each study. YZ and HC participated in the manuscript revision. All authors read and approved the final manuscript.

\section{Acknowledgement}

The authors would like to thank Dr.Qi Guang-zi for statistical work.

Received: 13 June 2013 Accepted: 30 September 2013

Published: 10 October 2013

\section{References}

1. American Cancer Society: Cancer Facts \& Figures 2012. Atlanta, GA: American Cancer Society; 2012.

2. Vergote I: Vlaamse werkgroep oncologische gynaecologie registratie 1998-2005 deel V ovarium. Tijdschr Geneesk 2006, 62:853-858.

3. Griffiths CT, Parker LM, Fuller AF: Role of cytoreductive surgical treatment in the management of advanced ovarian cancer. Cancer Treat Rep 1979, 63:235-240.

4. Hoskins WJ: Surgical staging and cytoreductive surgery of epithe-lial ovarian cancer. Cancer 1993, 71:1534-1540.

5. Hoskins WJ, Bundy BN, Thigpen JT, Omura GA: The influence of cytoreductive surgery on recurrence-free interval and survival in small-volume stage III epithelial ovarian cancer: a gynecologic oncology group study. Gynecol Oncol 1992, 47:159-166.

6. Chi DS, Musa F, Dao F, Zivanovic O, Sonoda Y, Leitao MM, Levine DA, Gardner GJ, Abu-Rustum NR, Barakat RR: An analysis of patients with bulky advanced stage ovarian, tubal, and peritoneal carcinoma treated with primary debulking surgery (PDS) during an identical time period as the randomized EORTC-NCIC trial of PDS vs neoadjuvant chemotherapy (NACT). Gynecol Oncol 2012, 124:10-14.

7. Vergote I, De Wever I, Tjalma W, Van Gramberen M, Decloedt J, van Dam P: Neoadjuvant chemotherapy or primary debulking surgery in advanced ovarian carcinoma: a retrospective analysis of 285 patients. Gynecol Oncol 1998, 71:431-436.
8. Inciura A, Simavicius A, Juozaityte E, Kurtinaitis J, Nadisauskiene R, Svedas E, Kajenas S: Comparison of adjuvant and neoadjuvant chemotherapy in the management of advanced ovarian cancer: a retrospective study of 574 patients. BMC Cancer 2006, 6:153.

9. Schwartz PE, Rutherford TJ, Chambers JT, Kohorn El, Thiel RP: Neoadjuvant chemotherapy for advanced ovarian cancer: long-term survival. Gynecol Oncol 1999, 72:93-99.

10. Morice P, Dubernard G, Rey A, Atallah D, Pautier P, Pomel C, Lhomme C, Duvillard $P$, Castaigne D: Results of interval debulking surgery compared with primary debulking surgery in advanced stage ovarian cancer. J Am Coll Surg 2003, 197:955-963.

11. Sehouli J, Savvatis K, Braicu El, Schmidt SC, Lichtenegger W, Fotopoulou C: Primary versus interval debulking surgery in advanced ovarian cancer: results from a systematic single-center analysis. Int I Gynecol Cancer 2010, 20:1331-1340.

12. Bristow RE, Chi DS: Platinum-based neoadjuvant chemotherapy and interval surgical cytoreduction for advanced ovarian cancer: a meta-analysis. Gynecol Oncol 2006, 103:1070-1076.

13. Morrison J, Haldar K, Kehoe S, Lawrie TA: Chemotherapy versus surgery for initial treatment in advanced ovarian epithelial cancer. Cochrane Library 2012, 8:CD005343.

14. Jacob JH, Gershenson DM, Morris M, Copeland L, Burke TW, Wharton JT: Neoadjuvant chemotherapy and interval debulking for advanced epithelial ovarian cancer. Gynecol Oncol 1991, 42:146-150.

15. Eisenkop SM, Spiretos NM, Montag TW, Nalick RH, Wang H: The impact subspecialty training on the management of advanced ovarian cancer. Gynacol Oncol 1992, 46:203-209.

16. Kayikcioglu F, Kose MF, Boran N, Caliskan E, Tulunay G: Neoadjuvant chemotherapy or primary surgery in advanced epithelial ovarian carcinoma. Int J Gynecol Cancer 2001, 11:466-470.

17. Surwit E, Childers J, Atlas I, Nour M, Hatch K, Hallum A, Alberts D: Neoadjuvant chemotherapy for advanced ovarian cancer. Int $J$ Gynecol Cancer 1996, 6:356-361.

18. Chambers JT, Chambers SK, Voynick IM, Schwartz PE: Neo-adjuvant chemotherapy in stage $\mathrm{X}$ ovarian carcinoma. Gynecol Oncol 1990, 37:327-331.

19. Schwartz PE, Chambers JT, Makuch R: Neoadjuvant chemotherapy for advanced ovarian cancer. Gynecol Oncol 1994, 53:33-37.

20. Hegazy MAF, Hegazi RAF, Elshafei MA, Setit AE, Elshamy MR, Eltatoongy M, Halim AA: Neoadjuvant chemotherapy versus primary surgery in advanced ovarian carcinoma. World J Surg Oncol 2005, 3:57.

21. Schwartz PE, Rutherford TJ, Chambers JT, Kohorn El, Thiel RP: Neoadjuvant chemotherapy for advanced ovarian cancer: long-term survival. Obstetr Gynecol Surv 1999, 54:379-381.

22. Morrison J, Swanton A, Collins S, Kehoe S: Chemotherapy versus surgery for initial treatment in advanced ovarian epithelial cancer. Cochrane Database Syst Rev 2007, 4:CD005343.

23. Morrison J, Haldar K, Kehoe S, Lawrie TA, et al: Chemotherapy versus surgery for initial treatment in advanced ovarian epithelial cancer. Cochrane Database Syst Rev 2012, 8:CD005343.

doi:10.1186/1477-7819-11-267

Cite this article as: Dai-yuan et al:: A meta-analysis: neoadjuvant chemotherapy versus primary surgery in ovarian carcinoma FIGO stagellI and IV. World Journal of Surgical Oncology 2013 11:267.

\section{Submit your next manuscript to BioMed Central and take full advantage of:}

- Convenient online submission

- Thorough peer review

- No space constraints or color figure charges

- Immediate publication on acceptance

- Inclusion in PubMed, CAS, Scopus and Google Scholar

- Research which is freely available for redistribution 